\title{
Optimizing Event Coverage in Theme Parks
}

\author{
Gürkan Solmaz and Damla Turgut \\ Department of Electrical Engineering and Computer Science \\ University of Central Florida \\ Email: \{gsolmaz,turgut\}@eecs.ucf.edu
}

\begin{abstract}
Theme parks can be modeled as geographical areas where large crowds of people move among different attractions. The operators of a theme park are interested in quickly and efficiently handling events occurring at various locations in the park. We propose a model which deploys a wireless network with mobile sinks to facilitate event coverage. The event coverage problem can be divided into two sub-problems: the static problem of mobile sink positioning and the dynamic problem of event handling decisions of the mobile sinks. For the mobile sink positioning problem we propose two strategies: crowd density based probability estimation (CDPE) and hot-spot based probability estimation (HSPE). For the event handling decision problem, we propose an approach which represents movement opportunities in the park as a graph with dynamically changing weights, and searches for the shortest path in this dynamic graph. The proposed approaches are simulated on scenarios which model the movement of the visitors using two sophisticated human mobility models.
\end{abstract}

\section{INTRODUCTION}

Theme parks are an important part of the entertainment industry and their successful operation can be a driver of local economic development. The operators of theme parks must solve a combination of technological and human challenges. Operationally, a theme park is a large geographical area where groups of visitors move between various attractions such as rides, restaurants, sights or rest areas. The operator of the theme park needs to manage the flow of visitors such that it maximizes their satisfaction by reducing the wait times at the various attractions. At the same time, the operator must be prepared to respond to events such as the operation of pickpockets, purse snatching, and disturbances caused by unruly visitors or medical emergencies. This leads to the challenge of event coverage [1]: the operator must acquire information about the events and decide how to handle them. Event handling and coverage is one of the major challenges in such environments due to inevitable security and emergency problems. In addition to the technological security measures, theme parks also deploy a large number of security employees, for some parks more than a thousand, walking on foot or riding bicycles [2]. We think that using of wireless sensor network with mobile sinks can increase the safety of the theme parks, and it may reduce the number of personnel needed for security.

In this paper we describe a method for optimizing event coverage using a wireless sensor network with mobile sinks. To achieve this, the theme park operator deploys two types of nodes in the geographical area of the park. Static sensor nodes are distributed throughout the theme park, passively sense the environment and transmit their observations to the mobile sinks. Mobile sinks collect data from the nodes, and move to the location of events. In general, we assume that the mobile sinks have more powerful networking and data processing capability, and they can act as actuators, actively resolving the events. Mobile sinks can be implemented, for instance, by security personnel carrying tablet computers and using electronic transportation devices. The movement of the mobile sinks is constrained by the capabilities of the mobility devices and the density of the visitors in various areas of the park.

We define the event coverage problem as the calculation of the movement of the mobile sinks for achieving an optimal coverage of events. Solutions to the event coverage problem must consider the a priori knowledge about the attractions and geography of the theme park and dynamic information about the movement of the visitors. Thus, the event coverage problem can be divided into two sub-problems: the positioning of the mobile sinks in the park in the absence of events and the event handling decisions of the mobile sinks.

The rest of the paper is organized as follows. We summarize related work in Section II. Section III describes the models of the landmarks of the theme park, the visitor movement and the wireless sensor network. Section IV describes the proposed solution strategies to the event coverage problem. The strategies are validated through a simulation study described in Section V. We conclude in Section VI.

\section{RELATED WORK}

In recent years, various studies focus on the use of mobile elements in wireless sensor networks. Mobility has significant effects on the performance of these networks and brings new research challenges for the existing problems such as data collection [3] and dissemination [4], relay node placement [5], [6], [7] path planning [8], latency [9], [10], lifetime maximization [11], [12], [13], [14], routing [15], [16], [17], [18] and security [19].

Let us briefly summarize the research on mobility models for network applications. Camp et al. [20] survey the mobility models used for ad hoc research and human mobility models that simulate the movement patterns of the mobile users. The human mobility models can be classified into synthetic [21] and trace-based [22] models mostly using GPS traces and Bluetooth connectivity. Synthetic models, which are defined on mathematical basis are widely used in the network simulations with human mobility since the amount of publicly available real-life data is limited. 
Among many, some of the literature on human mobility models also focus on using the models for performance evaluation of networks. Rhee et al. [23] show through the SLAW [24] model, the Levy walk features characterize the mobile network routing performance. ParkSim [25] by Vukadinovic et al. is a software tool for the simulation of wireless ad hoc networks and its mobility model is driven by the possible activities of the visitors. Using the model based on the GPS traces collected in a theme park, Vukadinovic et al. [25] observed the effects of human mobility on opportunistic message forwarding through wireless devices carried by the theme park visitors. Wang et al. [26] survey movement strategies for improving the performance of event coverage in wireless sensor networks.

Most research on wireless sensor networks with mobile elements (MEs) focus on settings such that large numbers of static sensor networks distributed in a large area and limited numbers of mobile sinks move between the sensor nodes to collect data. Data collection and management, transmission scheduling of the collected data, routing, and localization are major challenges in these networks. Di Francesco et al. [27] survey data collection schemes in WSNs with MEs, while Zhu et al. [28] survey communication and data management issues in mobile WSNs. Anastasi et al. [29] investigate data delivery to one or multiple MEs in the context of sparsely deployed sensor nodes. Turgut and Bölöni [30], [31] propose heuristic approaches for the transmission scheduling problem and compare the performances of each of the proposed strategies for WSNs with multiple mobile sinks. Bölöni and Turgut [32] suggest a decision-theoretic approach for the same problem. Luo et al. [33] show the benefits of routing towards a mobile sink approach for improving lifetime of WSNs in different scenarios. Furthermore, there are studies related to other types of WSNs with MEs, where the sensor nodes are also mobile [34], [35], [36] or the sensors are mobile and the sinks are static [37], [38]. There are also studies of using WSNs with MEs in 3D environments including aerial [39] and underground [40] scenarios. Erol-Kantarci et al. [41] survey distributed localization techniques used in mobile underwater acoustic sensor networks.

For managing positioning and mobility of the sink nodes, various approaches are proposed in the literature. Younis and Akkaya [42] survey techniques for careful node placement strategies used in WSN for effective optimization. Vincze et al. [43] use an approach similar to our crowd density based positioning strategy, for positioning multiple sinks optimally in a sensor network based on an electrostatic model by assigning positive or negative charges to sensor nodes according to their energy level, and positive charges to the sinks. In this study, the goal is to optimize energy usage of sensor nodes in the network. Wang et al. [44] survey the mobility management methods for mobile sensor networks and compare the methods in terms of their categories and characteristic features. Melodia et al. [45] suggest a location management scheme to handle the mobility of actors with minimal energy expenditure for the sensors, based on a hybrid strategy including location updates and location prediction. Vincze et al. [46] use an adaptive approach for sink mobility in event-driven multi-hop WSNs to minimize the maximum load on sensors and prolong the lifetime of the networks. They use an intruder movement model as the event model and propose two strategies to: a) minimize the sum of event distances and b) minimize the maximum energy consumption. Angelopoulos et al. [47] compare three coverage-adaptive random walks for fast sensory data collection. In Random Walk with Inertia, the mobile sink assigns probability to each directions and changes the probabilities by discovery of sensors. In Explore-and-Go Random Walk, the sink decides on moving in a straight line or changing direction based on a bias factor at each step. The last proposed walk is Curly Random Walk. In this approach, the sink traverses the network area starting from the center and expanding its mobility area by consecutive circular-like moves.

\section{System Model}

\section{A. The Operation of a Theme Park}

Theme parks are entertainment areas containing a number of attractions associated with specific geographic locations (landmarks). A visit in a theme park consists of three kinds of activities: (a) time spent moving between attractions, (b) time in the queue waiting for an attraction and (c) time spent visiting the attraction. Depending on the type of the attraction, different service models might exist. In restaurants, visitors are served individually, and the time spent at the table varies with the type of the meal and the customer's preferences. Other attractions, such as rides and theater performances, admit a limited number of visitors at a time, who remain there for the fixed duration of the show. There are also attractions which are available only at specific time-slots (open air concerts, parades). Visitors move among attractions on walking paths. In most cases, visitors can freely choose which attractions to visit next, but they are limited by the time it takes to walk from one to the other and the queues at the attraction.

In general, the goal of the visitors is to visit as many attractions as possible, with a higher preference for the more popular attractions. Although statistics of the visitors at various attractions and typical routes followed are available, there is a significant intra-day and day-by-day variation. The total number of visitors depends on the season, the day of the week and the time of the day. Unique entertainment events at specific time-slots can attract away the visitors from other attractions while meal times increase the traffic at restaurants. Long queues at one attraction might encourage visitors to try other attractions. Crowded walkways slow down the movement of the visitors. In conclusion, the movement of visitors in the theme park requires a complex model which takes into consideration the geography, the service models of the attractions, and the decisions of the visitors.

\section{B. Modeling the Movement of the Visitors}

As we have seen, the mobility of the visitors in a theme park depends strongly on the specific geography, attractions and schedules of the theme park, but it also contains many random elements due to arbitrary decisions taken by specific visitors. Thus, generic human mobility models do not predict well the visitor movement in a theme park: we need to use scenario 
specific models which take as input the a priori knowledge about the operation of the theme park.

We have developed the human mobility model [48] specifically to describe the movements of the visitors in theme parks. We start by generating fractal points representing the visiting points of the human walkers. The dense regions where visitors spend most of their time, corresponding to popular attractions are found using the DBScan [49]. Figure 1 shows the initial steps of the model. In the first step, 2000 fractal points synthetically generated, and in the second step, the 15 clusters of fractal points found by DBScan. These clusters correspond to the attractions in a theme park. The nonclustered points in the model represent points visited by human walkers outside the attractions. The number of clusters (i.e. 15) and the percentage of non-clustered fractal points (i.e. 10\%) are two parameters of the model, which are used in the second step. We call the geographic location of the clusters and nonclustered points landmarks.

To model the behavior of the visitors waiting in queues and visiting attractions, we associate with each attraction (cluster) a queue model. The nature of the queue depends on the type of attraction. For instance, food stalls are modeled by M/M/1 queues, representing the fact that the arrival happens independently, and the service time is variable. Rides are modeled by $\mathrm{M} / \mathrm{D} / \mathrm{n}$ queues, as their service time is deterministic and they can serve $\mathrm{n}$ customers at a time. Live shows, where the time of the event is not deterministic and visitors can leave at any moment are modeled as $\mathrm{M} / \mathrm{M} / \mathrm{n}$ queues. We call the non-clustered fractal points as the noise points.

Figure 2 illustrates the attractions (queues), the nonclustered visiting points (noise points), and the positions of 200 visitors in the park. The queues, which are generated using the clustered points, are represented by squares. The non-clustered visiting points and visitors are shown by small dots and circles respectively. Each queue is labeled with its queue type: major rides (RD), medium-sized rides (M-RD), live shows (LS), and restaurants (RT).

The algorithm for deciding the next destination [48] chooses the next target from the set of unvisited locations based on probabilities which favor the closer locations and locations which correspond to more popular attractions. Visitors try to minimize the Euclidean distances traveled between popular attractions and visiting points. On the other hand, all selected attractions and visiting points have the chance to be selected as next destinations.

Visiting an attraction is physically represented by the visitor moving to a random point inside the region of the attraction (this can be, for instance, the location from where the visitor watches a show). Each queue has a service rate, the number of visitors per service, and capacity. The waiting time of a visitor in a queue depends on the number of visitors already waiting in the queue ahead of the visitor, the service rate, and the number of visitors per service of the queue. On the other hand, when a visitor goes to a noise point, the visitor spends time in that location for a waiting time generated randomly by truncated Pareto distribution.

In the model, the visitors of theme parks are represented by mobile nodes. The mobile nodes have states representing their current activities during their hangout times in the landmark: initial, inQueue, moving, inNoisePoint and removed. All the mobile nodes are in the initial state at the beginning of the mobility simulation. The node is in inQueue state if it is waiting in the queue or is serviced by the queue. Thus, waiting time in the queue for the mobile nodes is the sum of waiting time and service time. Furthermore, if a queue is on its full capacity whenever a mobile node arrives, the node bypasses the queue without marking the queue as visited and selects another destination to visit. If a node selects a noise point as the next destination, it changes its state to inNoisePoint whenever it arrives to the noise point and waits for the generated waiting time. There are two different waiting states in the model to differentiate the queues and the noise points. After the waiting time of a node in a queue or in a noise point passes, the node decides a new destination, changes its state to moving, and starts to move to the new destination. Finally, whenever the hangout time of a mobile node in a landmark passes, the state of a mobile node becomes removed.

Let us describe the pre-planning phase of the visitors. In the initial state, each node decides its own hangout time for the landmark. The hangout time is generated randomly by exponential distribution. Based on the hangout time, each visitor selects a subset from the set of all queues and marks the queues in the subset as planned to be visited. The number of queues to be visited (size of the subset) is proportional with the hangout time. Having a plan in the initial phase does not guarantee that all the selected subset of queues is going to be visited, but each mobile node aims to visit as many of them as possible. During the simulation, a mobile node can behave non-deterministically by selecting a noise point and spend time there or by selecting a queue which is not the closest one as the next destination. The queues, on the other hand, have a deterministic behavior since they have specified parameters such as expected service time, number of mobile nodes serviced at each service time, and capacity.

To serve as a basis of comparison, our simulations also use an alternative human mobility model, the self-similar least action walk (SLAW) [24]. The SLAW model provides an effective strategy for representing social contexts present among people sharing common interests or those in a single community such as a university campus, companies and theme parks. In this model, the social contexts of common gathering places are represented by fractal points and heavy-tail flights on top of these fractal points. Both models used in our simulations are validated by GPS traces collected from theme park visitors.

\section{Wireless Sensor Network Model}

In the following we describe the model of a wireless sensor network with mobile sinks specifically designed to allow the operator of the theme park to efficiently and promptly handle events. The sensor network consists of two types of nodes.

Static sensor nodes are deployed throughout the theme park. Their capabilities are limited to sensing events occurring in their physical vicinity, and the transmission of their observations to the mobile sinks using hop-by-hop transmissions. The 

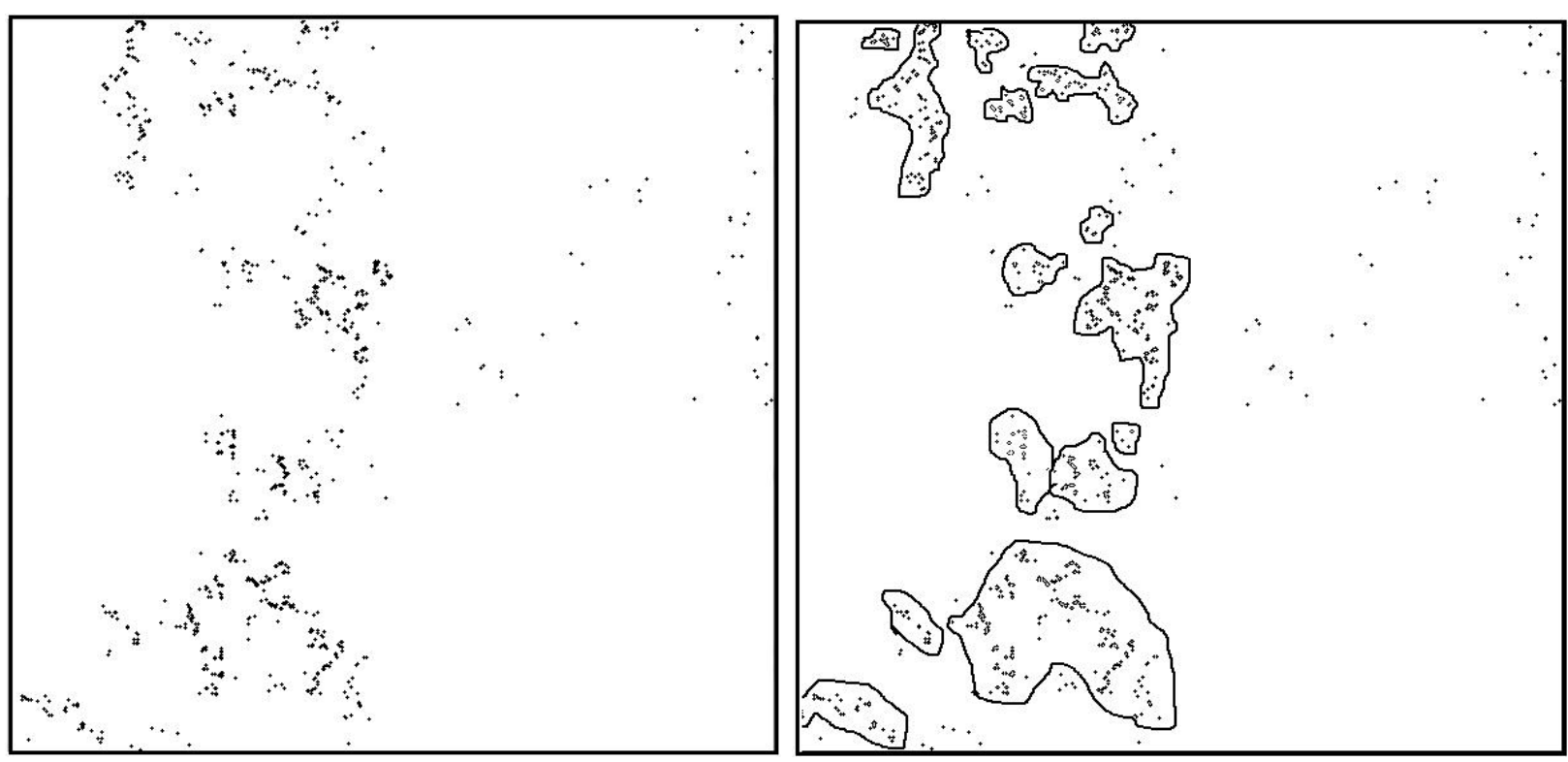

Fig. 1. Generating a model of the visitor mobility. Left: 2000 fractal points representing visitor destinations, right: 15 clusters representing popular areas in the theme park.

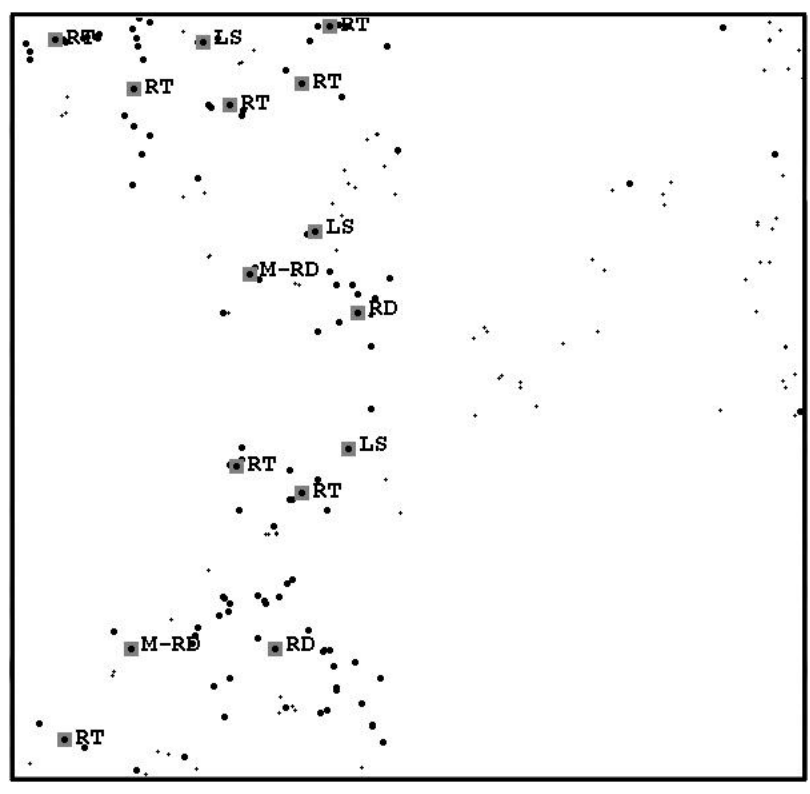

Fig. 2. Queues, non-clustered points and 200 visitors in the theme park.

static nodes are passive observers, they cannot take actions to handle events. Sensor nodes may stay idle when there is no event or they may sense environmental data for regular monitoring purposes. We assume that static nodes are deployed uniformly and randomly in the geographical area of the theme park. In this study we focus on mobile sink positioning and the selection of the best sink for event coverage. Naturally, for a complete system, an appropriate routing protocol for WSNs with mobile sinks must be chosen [50].

Mobile sinks are nodes with the ability to receive information from the static nodes, move to the location of the events and take active steps towards handling the event. We assume that every event has an associated timeout, a window of time within which the event can be handled. If no mobile sink reached the event before the timeout, we consider that the system missed the event. The objective of the system is to minimize the time from the detection of an event to the time it is handled. We assume that mobile sinks have the ability to share among each other their locations, computation results (decisions) and event information. highightThe information is assumed to be shared directly via wireless communication between the mobile sinks whenever possible or transmission via sensor nodes between them. Moreover, we assume that the conditions of the roads and attractions in terms of the estimated traveled time and crowdedness are shared between the mobile sinks in the same way. For instance, a mobile sink computes the estimated travel time according to the conditions of the road and share this information. Note that this information can be shared by a control center which globally estimates the number of people in the attractions and roads if the mobile sinks are accessible. A possible real-world implementation of a mobile sink can be a security guard driving a personal electric transportation vehicle (e.g. Segway Patroller [51]) with a tablet computer attached to the vehicle.

Between handling events, mobile sinks must be positioned such that the handling time of future events is minimized. The number of mobile sinks deployed can change during the day (for instance, due to equipment failure or their operators taking a break). The position of the mobile sinks must be adapted to the number of currently active units.

Mobile sinks have a maximum speed determined by the technology used. Furthermore, their speed is limited by environmental obstacles, such as the density of the crowd on the paths. The mobile sinks need to plan their movement with a consideration of such slowdowns; the shortest path might not necessarily be the fastest way to reach the destination. 


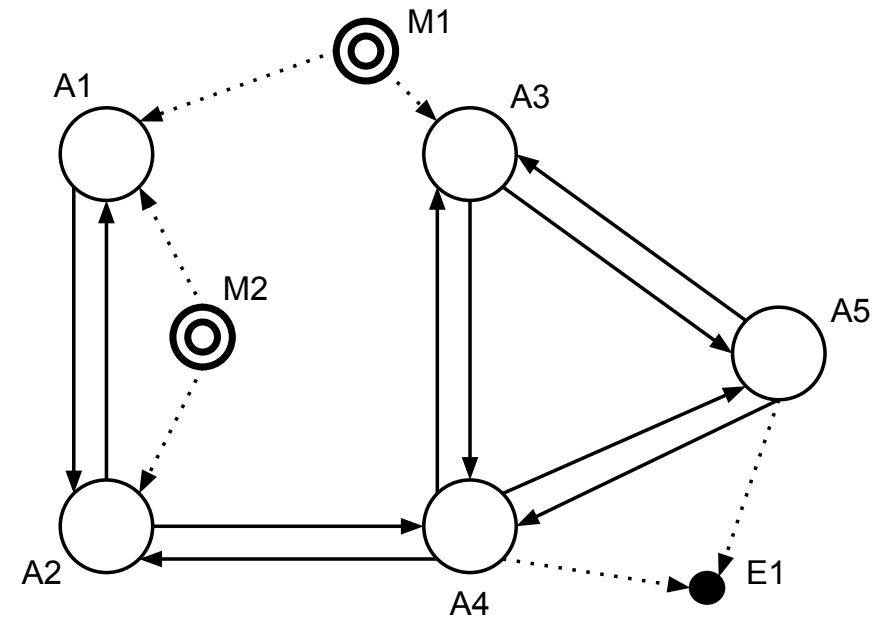

Fig. 3. The dynamic directed graph model.

\section{Event Model}

Let us now consider a model of the occurrence of the events in the theme park environment. We model the start times of the events through a Poisson process, while the location of an event follows one of the following three random spatial distributions:

- Uniform random event distribution: events occur close to one of the attractions, with all attractions having the same probability to host the next event.

- Biased event distribution: events are distributed according to initial probabilities of attractions, with events more often occurring at popular attractions.

- Scenario-specific event distribution: certain attractions have a significantly higher probability of an event to occur. This might be caused, for instance, by insufficient safety or security measures.

The distribution of events may change in time. For instance, new security and safety measures may change the distribution of the events from scenario-specific to biased event distribution. The wireless sensor network must adapt to all of the three cases using effective strategies.

\section{E. Dynamic Directed Graph Model}

In the previous sections we introduced the various entities which contribute to the description of the state of the theme park augmented with a sensor network: attractions, movement paths, visitors, mobile sinks and events. In this section, we combine these in a formal model which supports the algorithms implementing the decisions of the mobile sinks. The model is implemented as a directed weighted graph. The nodes of the graphs represent the locations of the attractions, the current locations of the mobile sinks and the locations where the events happened. The edges represent available movement paths. The weights of the edges represent the time estimate for a mobile sink to travel between the nodes. For instance, to estimate the time for a mobile sink to reach an event, we need to find the shortest path from the current location of the sink to the event. In order to simplify the graph, we will not include edges which are irrelevant from the point of view of the movement of the mobile sinks (for instance, edges from the attractions and events to the mobile sink locations).

Figure 3 shows an example graph where $A 1 \ldots A 5$ are attractions, $M 1$ and $M 2$ are mobile sinks and $E 1$ is an event. The graph is dynamic in the sense that some of its components depend on the current circumstances of the theme park. The attractions and their connecting links are permanent features of the graph. However, the weight of the edges connecting the attractions varies with the population of the visitors on these paths. The nodes describing the current mobile sink locations and events, as well as their edges must be generated dynamically based on the current situation.

Attraction nodes are marked with the probabilities of event occurrences. The initial probability values are set according to the estimated number of visitors for each attraction. This estimation can be based on the previous observations and statistics of the attractions. In our model we use fractal points to estimate the average number of visitors in an attraction. The distribution of the fractal points corresponds to the popular areas in the theme park. The initial probability values for the attraction nodes are computed as follows:

$$
P(A i)=\frac{F(A i)}{F(A 1)+F(A 2)+\ldots+F(A n)}
$$

where $P(A i)$ is the probability value of an attraction $A i$, $F(A i)$ is the number of fractal points in the cluster corresponding to the attraction $A i$ and $n$ is the number of attractions in the landmark. The sum of the probability values of all attractions is equal to 1. Clearly, this is a rough estimate, since we do not consider some properties of the theme park model, such as the capacities of the attractions, statuses of the queues, and so on. These estimates are only used for the initial values of the attraction probabilities and they are updated dynamically. The update mechanisms for probability values are described in the following section in detail.

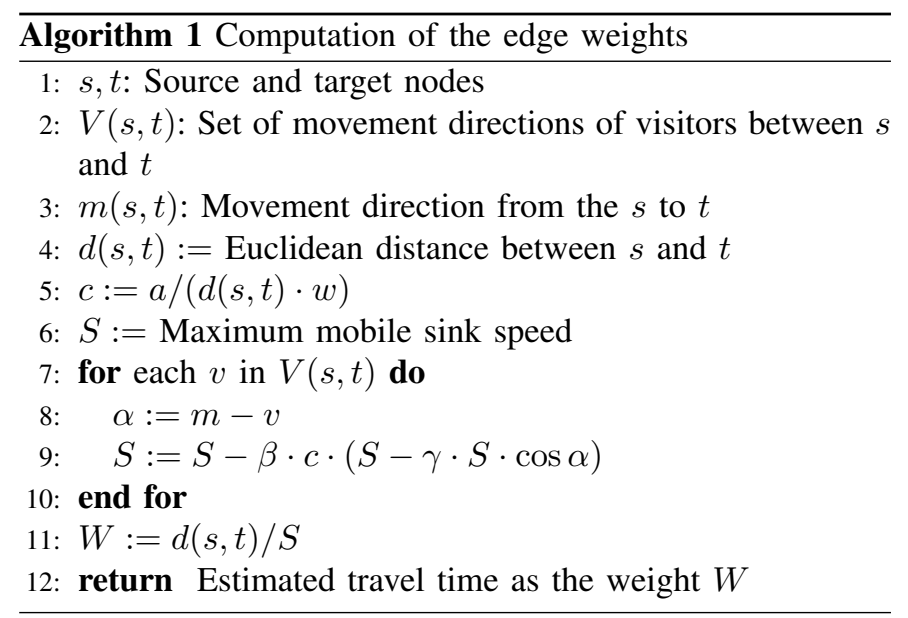

The most challenging part of the graph construction is the calculation of the weights, which represent estimates of the travel time of the mobile sinks on specific paths. Intuitively, if the path is empty, the mobile sink can move with its maximum speed - in our case, the $12.5 \mathrm{mph}$ speed of the Segway Patroller 


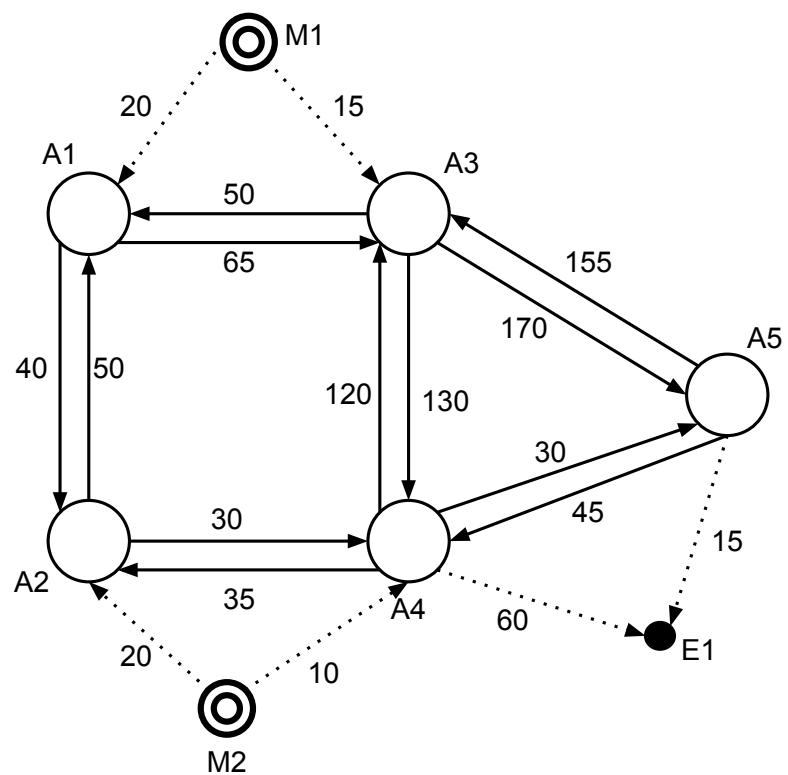

Fig. 4. The dynamic edge weights created when an event $E 1$ happens.

device. However, if the mobile sink shares the road with visitors, it needs to slow down to avoid collisions. The higher the density of the visitors on the road, the more significant the slowdown. Finally, visitors moving in the same direction as the sink trigger less slowdown than visitors moving in the opposite direction - which means that the weights attached to opposite edges can be different. Thus, calculating the weights of the graph requires estimates of the density and the movement of the visitors, and information provided by the static sensor nodes.

The algorithm for calculating the weights is described in Algorithm 1. The visitor density $c$ is the number $a$ of visitors currently located in the road divided by the area of the road. The area of the road is equal to $d(s, t) \cdot w$ where $w$ is the width. $\alpha$ is the direction difference between the sink and a visitor and $\gamma \in(0,1]$ is the constant for adjusting the effect of the direction $\alpha$. The parameter $\beta$ allows us to scale the impact of each visitor on the speed of the mobile sink. This parameter allows us to approximate the effect of larger crowds than the ones we are effectively simulating.

An example of the results of this algorithm are shown in Figure 4. In this figure, the edges drawn with dotted lines are the dynamic edges: the edges updated through the movement of the mobile sinks and the edges connecting the new event $E 1$ to the closest attractions. We use an adjacency matrix to represent the graph in the implemented algorithm. Figure 5 shows the adjacency matrix associated with the graph in Figure 4 with the value $x$ in the cells showing no edge between the two nodes.

\section{Solving the Event Coverage Problem}

Solving the event coverage problem involves resolving the events occurring during the operation of the theme park as efficiently as possible. This involves two sub-problems. The mobile sink positioning problem chooses the location of the

\begin{tabular}{|c|c|c|c|c|c|c|c|c|}
\hline & A1 & A2 & A3 & A4 & A5 & M1 & M2 & E1 \\
\hline A1 & $\times$ & 40 & 65 & $\times$ & $\times$ & $\times$ & $\times$ & $\times$ \\
\hline A2 & 50 & $\times$ & $\times$ & 30 & $\times$ & $\times$ & $\times$ & $\times$ \\
\hline A3 & 50 & $\times$ & $\times$ & 130 & 170 & $\times$ & $\times$ & $\times$ \\
\hline A4 & $\times$ & 35 & 120 & $\times$ & 30 & $\times$ & $\times$ & 60 \\
\hline A5 & $\times$ & $\times$ & 155 & 45 & $\times$ & $\times$ & $\times$ & 15 \\
\hline M1 & 20 & $\times$ & 15 & $\times$ & $\times$ & $\times$ & $\times$ & $\times$ \\
\hline M2 & $\times$ & 20 & $\times$ & 10 & $\times$ & $\times$ & $\times$ & $\times$ \\
\hline E1 & $\times$ & $\times$ & $\times$ & $\times$ & $\times$ & $\times$ & $\times$ & $\times$ \\
\hline
\end{tabular}

Fig. 5. The adjacency matrix with dynamic edge weight values, corresponds to the graph model in Figure 4.

mobile sinks in the absence of any event. The goal is to distribute the mobile sinks in such a way that when events happen, there will be a sink nearby which can quickly reach the location of the event. Naturally, in order to find such a solution, we can rely on our statistical knowledge about the locations where events are likely to occur, and about the time to reach specific locations from the current location of the sink. The second sub-problem relates to the handling of events. Once an event occurred, we need to decide which mobile sink will handle the event, and which path it will follow to the location of the event.

For both sub-problems, we assume that the mobile sinks know the location of each other and the events each of them are handling. The dynamic model of the theme park, in the form of the weighted directed graph described in the previous section, is shared by all the mobile sinks.

\section{A. Mobile Sink Positioning}

We propose a mobile sink positioning algorithm which is based on estimating the probability of events happening at various locations, then assigning the mobile sinks in such a way that they are grouped towards the most likely locations of events. Algorithm 2 shows how to update the location of the mobile sinks. We assume that the mobile sinks are stationed at attractions, $A(m)$ representing the current attraction of the mobile sink $m$. At each step, the mobile sink must make a decision to either stay at its current attraction, or to move to a neighboring attraction, if that attraction has a higher probability of an event to occur and is not already occupied by another mobile sink.

This algorithm, taken as a high level framework can be customized in a number of ways. First, we can choose different definitions of the neighbors of a node: it can represent either a distance of a single edge in the graph, or it can represent all the attractions which are within a distance threshold. Another way in which the algorithm can be customized is by adapting the definition of an occupied attraction: for very high event 
probabilities, we can allow for more than one mobile sink to be stationed at the same attraction.

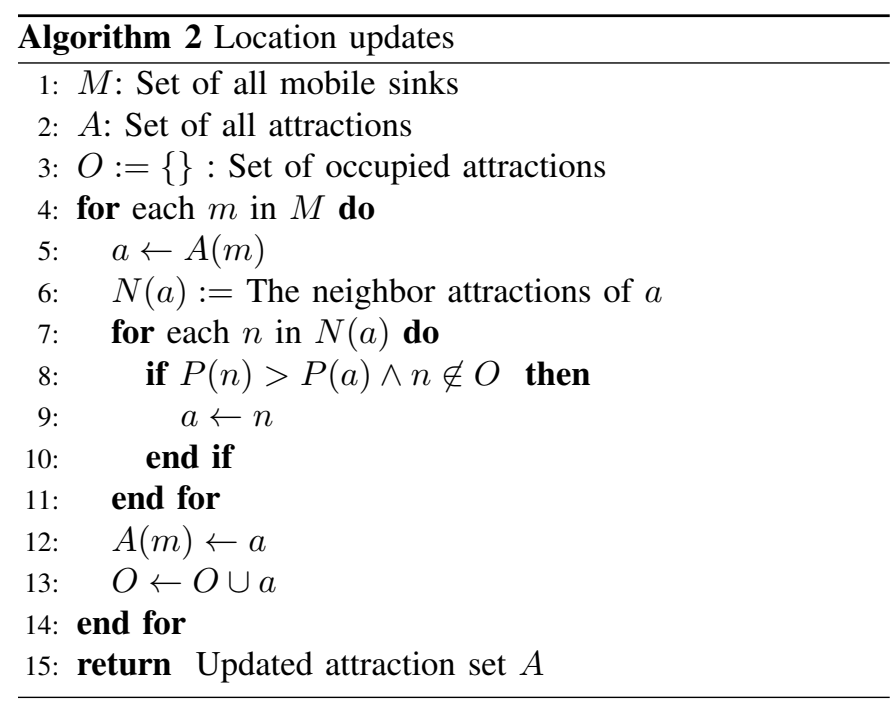

The positioning algorithm requires an estimate of the probability of future events. In the following we propose two such estimation techniques, based on different assumptions about the nature of events: a crowd density based and a hot-spot based probability estimation technique.

The crowd density based probability estimation (CDPE) assumes that the number of events at an attraction is proportional to the number of visitors. The number of visitors at the attraction is estimated by the sensor nodes.

CDPE assumes that each visitor contributes equally to the probability of an event. However, experience shows that certain locations are more likely to have events (possibly due to the nature of the attraction). Furthermore, events are frequently clustered into hot-spots (possibly, due to a common, but hidden cause).

The hot-spot based probability estimation (HSPE) takes into consideration the history of events at specific locations. The occurrence of an event increases the probability another event will occur at the same attraction. The equation below shows the formula used for updates done after an event occurs. For each event $e$, the probability of an event at attraction $A i$ increases if the event happened in that attraction. The magnitude of the increase is calculated by multiplying the priority of the event $\rho \in 1 \ldots 5$ with the adaptivity value constant $\delta($ e.g. $\delta=0.05)$. It decreases for all other attractions by the same value divided by the number of other attractions $(n-1)$.

$$
P(A i)= \begin{cases}P(A i)+\rho \cdot \delta & \text { if } e \text { occurred in } A i \\ P(A i)-\frac{\rho \cdot \delta}{n-1} & \text { otherwise }\end{cases}
$$

\section{B. Event Handling}

The second sub-problem we are considering is that of event handling. Let us assume that the mobile sinks are positioned using one of the algorithms from the previous section, and an event $E 1$ occurs. The event handling algorithm needs to decide

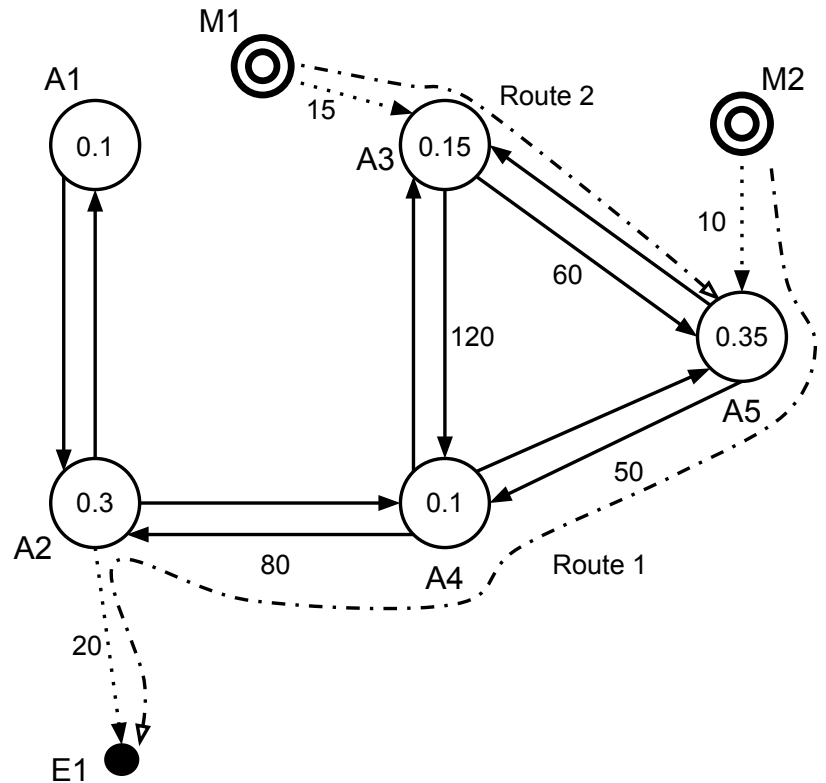

Fig. 6. An example mobile sink selection and covering of the attractions.

(a) which mobile sink will handle the event and (b) which path it will follow when moving to the event's location.

We have developed two algorithms, corresponding to different levels of information available about the state of the theme park. If the information collected from the sensor nodes allow us to estimate the density and movement directions of the visitors on the paths, we can deploy the Fastest Responder (FR) strategy, which assigns to every event the mobile sink which can get there the fastest. To implement this, we create the dynamic graph of the current state of the node using Algorithm 1. The weight of the edges correspond to time needed to traverse them. On this time-weighted graph, we calculate the shortest path from every mobile sink node to the event node. The mobile sink which will be assigned to the event will be the one which is at the shortest distance in this graph. Note that the fastest responder might not be the one which is physically the closest.

Figure 6 illustrates an example run of this algorithm. In this figure, numbers near the arrows represent the edge weights while numbers inside the graph nodes are probabilities of attractions. There are two routes, Route 1 and Route 2, for the two sinks to follow. In this example, the mobile sink $M 2$ is responsible for the attraction $A 5$ and the other mobile sink $M 1$ is responsible for $A 3$ at the beginning. When the event $E 1$ occurs, the mobile sink $M 2$ is selected to handle the event $E 1$ since the shortest path of $M 2$ is 160 while the shortest path of $M 1$ is $220 . M 2$ follows Route 1 to reach the event. The movement of $M 2$ to handle the event, however, also triggers changes in the static positioning of the other mobile sinks. The probability of attraction $A 5$ is $P(A 5)=0.35$ while the probability of $A 3$ is $P(A 3)=0.15$. Therefore the mobile sink $M 1$ changes its position to cover the attraction $A 5$ with higher probability by following the Route 2 , the shortest path from its current location to the attraction $A 5$.

For the case when the monitoring of the movements of 
visitors is not possible, we use the Closest Sink (CS) strategy. In this strategy, the shortest path algorithm is used on a static graph, where the edge weights correspond to Euclidean distances rather than in travel times.

\section{Simulation Study}

\section{A. Simulation setup}

In the following we describe a series of simulation experiments which study the performance of the proposed event coverage algorithms.

Let us start with describing the simulation setup. We are considering a rectangular theme park of size $1000 \mathrm{~m}$ x $1000 \mathrm{~m}$, with 15 geographically distributed attractions, whose locations have been determined using a fractal point model with 1000 fractal points. Between these attractions, we created the paths in two ways: (a) by connecting all attractions with a vertex degree of 4 or higher and (b) by adding paths between random attractions until the graph density reaches 0.7 .

We have considered 500 visitors who move around and visit attractions in the park. We generated two different datasets of visitor movement based on the theme park mobility model (TP) [48], and the SLAW movement model [24]. Each dataset contains trajectory files for 10 hours of mobility with a 10 seconds sampling time. The movement speed of visitors had been set to $1 \mathrm{~m} / \mathrm{s}$. According to Wanhill [52], the attractions in a theme park are defined by queuing models and their percentages are distributed as given in Table I [48].

TABLE I

ATTRACTION PERCENTAGES

\begin{tabular}{|l|l|l|}
\hline Attraction & Queue model & Percentage \\
\hline Main rides (RD) & $\mathrm{M} / \mathrm{D} / \mathrm{n}$ & $17 \%$ \\
\hline Medium-size rides (M-RD) & $\mathrm{M} / \mathrm{D} / \mathrm{n}$ & $56 \%$ \\
\hline Restaurants (RT) & $\mathrm{M} / \mathrm{M} / 1$ & $17 \%$ \\
\hline Live shows (LS) & $\mathrm{M} / \mathrm{M} / \mathrm{n}$ & $10 \%$ \\
\hline
\end{tabular}

The theme park contains from 1 to 20 mobile sinks with a maximum speed of $5.58 \mathrm{~m} / \mathrm{sec}$. The location update time for adaptive mobile sinks was set to 30 minutes. Sensor nodes are assumed to transmit the information of events to the mobile sinks whenever an event occurs, and mobile sinks share the current conditions of the dynamic theme park graph in the specified location update times.

Let us now discuss the generation of the events. We need to consider three aspects: the location of the events, their starting times, and their active duration. The location of the events had been created using one of the three event distribution algorithms described in Section III-D. The arrivals of the events are modeled with a Poisson process with an average arrival rate of 30 events / hour. The active time of the events is randomly distributed in the range of 60 to 300 seconds.

Throughout the simulation scenarios, we measured two performance metrics. The average event handling time is the travel time for the selected mobile sink to reach an event. This also includes situations where the sink reaches the location of the event after the event's active time expired. The handling success ratio is the fraction of the times the sink reaches the event before its active time had expired.

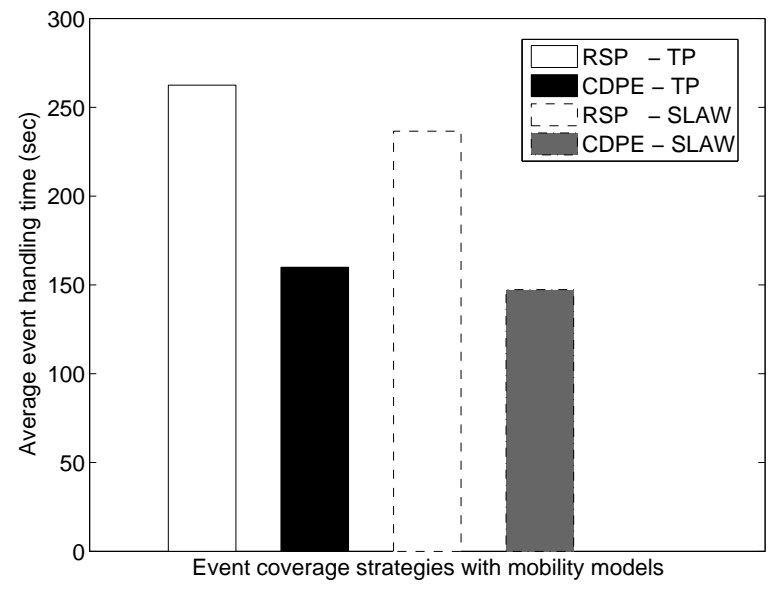

Fig. 7. Average event handling times for CDPE vs. random sink positioning.

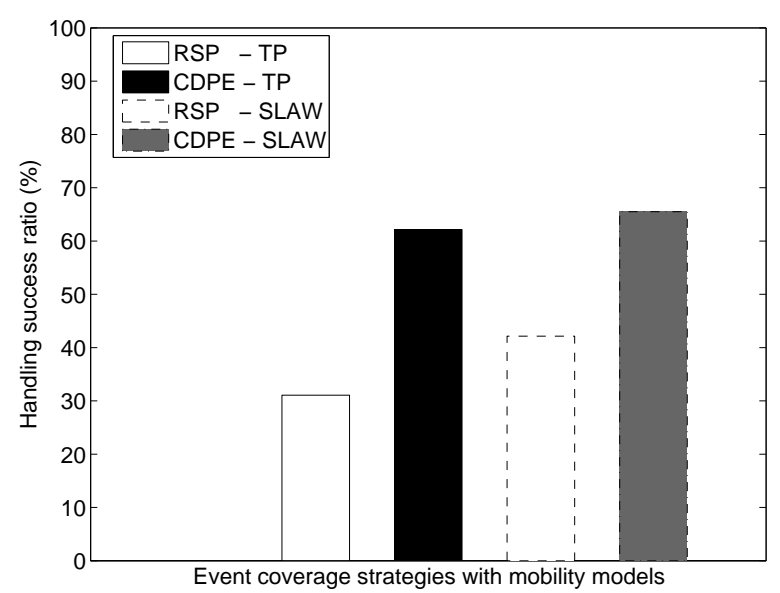

Fig. 8. Handling success ratios for CDPE vs. random sink positioning.

\section{B. Simulation results}

1) Performance of CDPE: In the first of set of experiments, we evaluated the performance of the crowd density based probability estimation (CDPE) strategy. As a comparison baseline, we used the random sink positioning (RSP) strategy, where the sinks are deployed uniformly and randomly in the theme park. The experiments considered 5 sinks. The location of the events was generated using the biased event distribution. For both positioning strategies, the fastest responder (FR) strategy was used to handle the events. The experiments were repeated with the visitor movements generated from the TP and the SLAW mobility models respectively.

Figure 7 shows the average event handling times for the combinations of positioning strategies and visitor mobility models. For both mobility models, the CDPE strategy clearly outperforms the RSP strategy, in average providing a $40 \%$ faster event handling time. Incidentally, the handling times are about $8 \%$ longer for the TP mobility model compared to SLAW. This difference justifies the development of theme park specific mobility models (compared to general purpose 


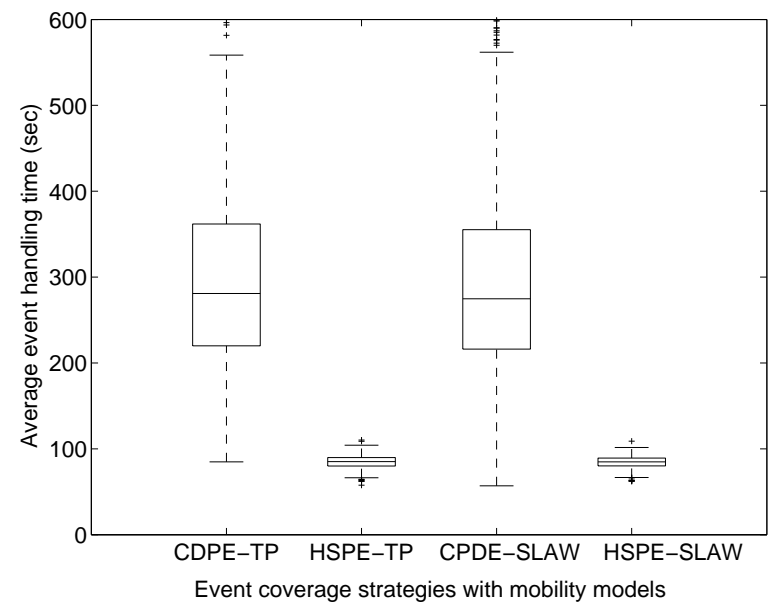

Fig. 9. Average event handling times for sink positioning by HSPE vs. CDPE.

mobility models).

The faster response times of the CDPE strategy are also reflected in the higher success ratio as shown in Figure 8. CDPE can handle about $65 \%$ of the events within their deadlines, while RSP handles $30 \%$ for the TP mobility model and about $40 \%$ for SLAW. We notice that the binary nature of the event handling success magnifies the difference between the success ratios of RSP for the TP and SLAW models respectively.

2) Performance of HSPE: We designed the HSPE positioning technique to handle scenarios where the events occur more frequently at certain attractions (the event hot spots). To study the performance of HSPE, we generated scenarios where three attractions are randomly selected to become hot spots. Our hypothesis is that under these circumstances, HSPE will outperform CDPE.

The simulation was run using 3 sinks and the FR responder strategy. We repeated the simulation for both the TP and SLAW mobility models. Figures 9 and 10 show the average event handling times and handling success ratios of 500 experiments for each result. We decided to present the results using box plots to illustrate the variation of the performances. The results show that the HSPE algorithm highly outperforms CDPE in these scenarios for both performance metrics. In addition, HSPE shows very little variation in the performance. In contrast, CDPE shows a significant performance variation.

The performance increase is attributable to the additional information exploited by the HSPE algorithm about historical event locations. As a note, as HSPE will behave the same as the CDPE strategy in the absence of hot spots, we recommend the use of the HSPE strategy for all deployments.

In the experiments shown in Figures 9 and 10, we considered the specific case where the number of hot spots matched the number of mobile sinks. While this might appear arbitrary, it is, in fact, a good deployment strategy to have at least as many mobile sinks as the number of hot spots.

In the following, we compared performance of the HSPE strategy against sink positioning by CDPE for various numbers

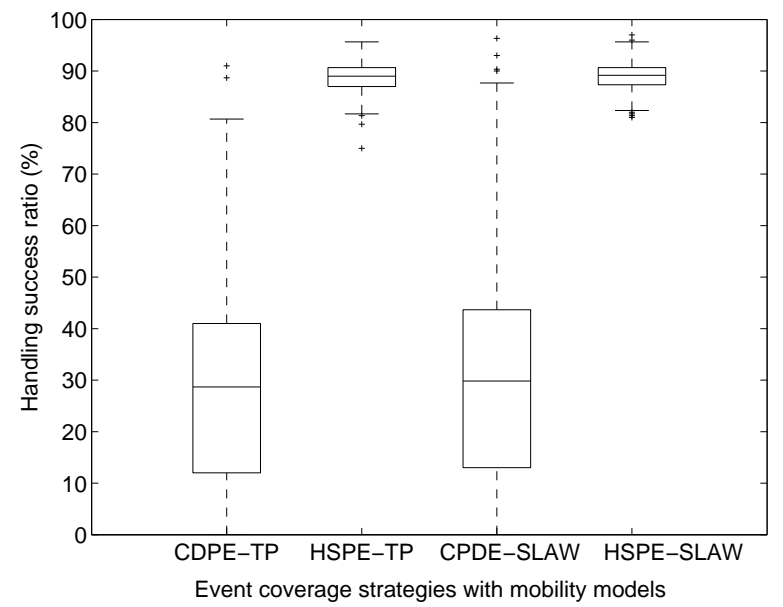

Fig. 10. Handling success ratio for HSPE vs. CDPE positioning strategies.

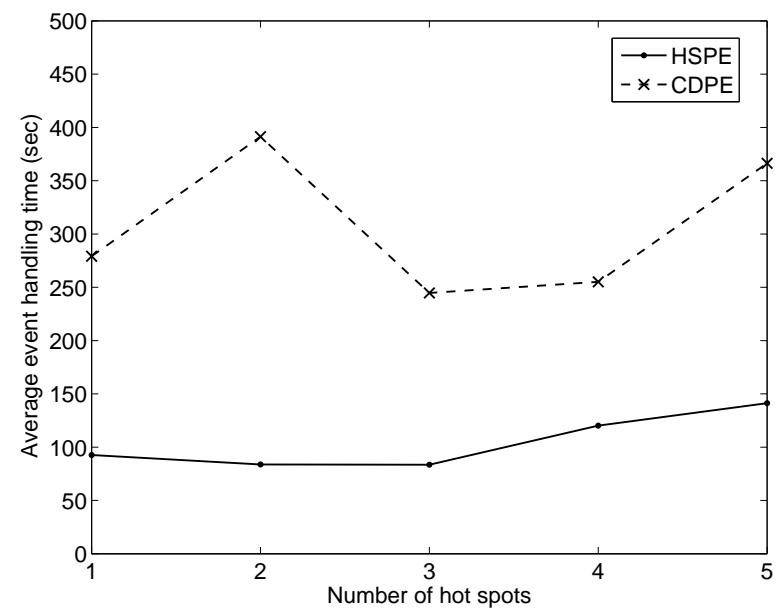

Fig. 11. Average event handling times for HSPE vs. RSP for 1 to 5 hot spots.

of hot spots. 3 mobile sinks are used for handling events created randomly from 1 to 5 hot spots. As before, we used the FR event handling strategy, and generated events with the TP mobility model.

Figure 11 shows the average event handling times and Figure 12 shows the handling success ratios for the two strategies. Overall, HSPE significantly outperforms CDPE for all the scenarios. In general, HSPE obtains its best performance when the number of sinks is equal to those of the hot spots. Once the number of hot spots exceed the number of sinks, the average event handling time increases and correspondingly, the handling success ratio decreases. CDPE on the other hand not only has a lower performance, but the performance varies with the number of hot spots significantly and without a clear pattern. The reason behind this erratic behavior is that for this scenario CDPE had simply made the wrong assumptions: it assumed that the events follow the crowd distribution, while in reality, they were concentrated in hot spots. 


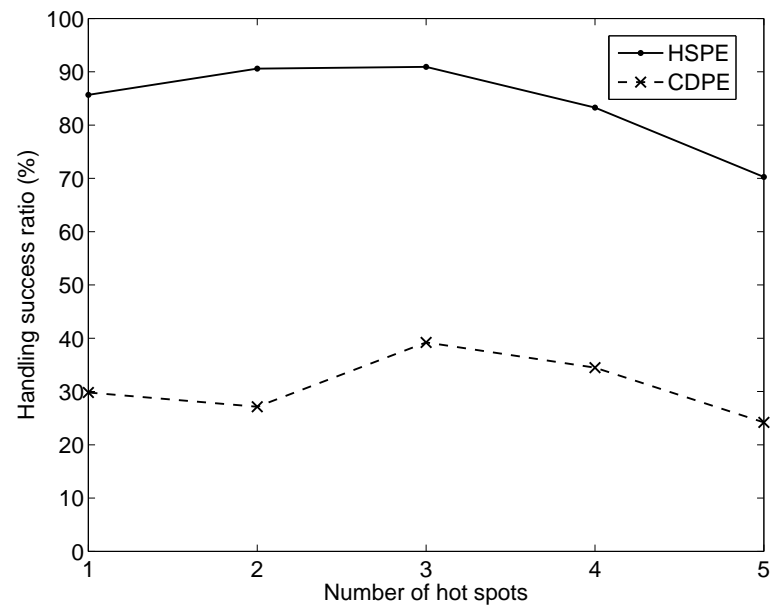

Fig. 12. Handling success ratio for HSPE vs. CDPE for 1 to 5 hot spots.

3) Performance of CDPE and HSPE Function of the Number of Mobile Sinks: In general, we expect that increasing the number of mobile sinks improves the performance of the event handling, because if enough mobile sinks are spread around in the area, no matter where the event occurs, some mobile sink will be close enough to reach it fast. However, adding sinks is a significant expense, thus it is important to investigate the point where adding more mobile sinks provides minimal advantages.

We have run a series of experiments using the HSPE, CDPE and RSP positioning algorithms. We used the TP mobility model and the FR strategy to handle the events. The number of mobile sinks was varied between 1 and 20. The results for the event handling time is shown in Figure 13 while for the handling success ratio in Figure 14. As expected, for all the algorithms the performance increases with the number of sinks deployed, and the increase is especially fast when moving from 1 to 2 and from then on to 3 sinks. However, the performance eventually reaches a plateau: even for the best performing HSPE algorithm, no additional increase appears to decrease the average handling time below 70 seconds, nor push the handling success ratio above $93 \%$.

The comparative performance of the algorithms, is as expected: HSPE is the best, followed by CDPE and RSP. This holds true for all sink numbers (a single outlier aside). More important however, is the difference in the level of the plateaus. While HSPE reaches a plateau at around 93\%, CDPE and RSP cannot be pushed above $80 \%$ regardless of the number of mobile sinks deployed. In fact, HSPE can achieve better performance with 6 mobile sinks, than the other algorithms would achieve with 20 .

The graphs in Figures 13 and 14 can also be used for efficient allocation of human resources. It is not economically justified to add additional mobile sinks after reaching the plateau. With the assumptions we considered, for HSPE there are very few benefits to gain from adding more than 10 sinks, and none in adding more than 14 . These values might change with different assumptions.

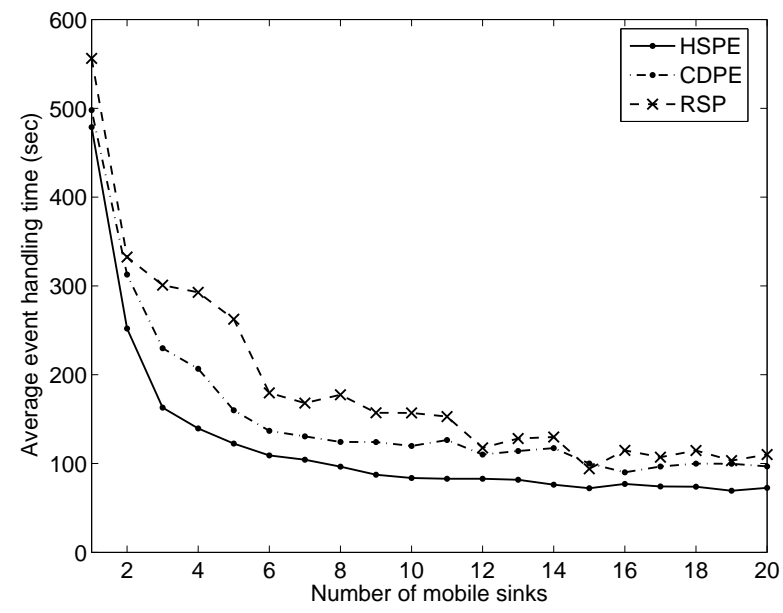

Fig. 13. Effect of multiple mobile sinks on event handling time for the HSPE, CDPE and RSP algorithms

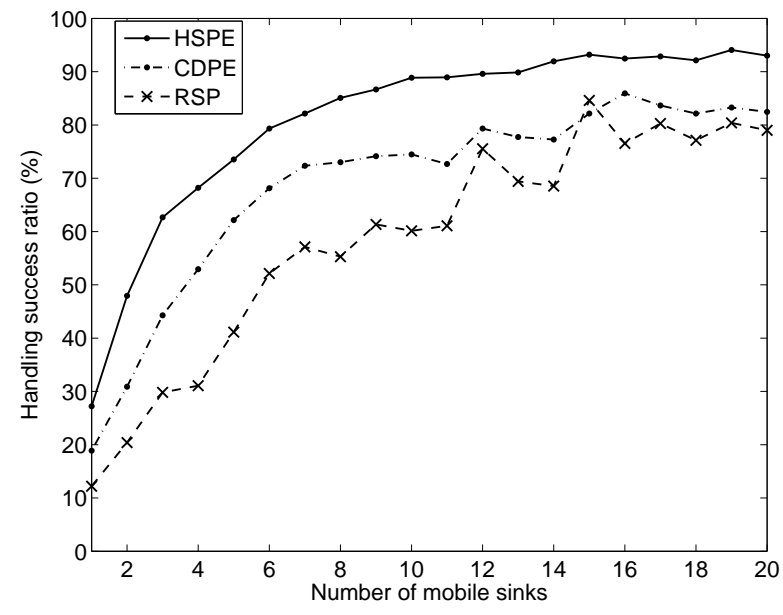

Fig. 14. Effect of multiple mobile sinks on handling success ratio for the HSPE, CDPE and RSP algorithms

4) Performance of Event Handling Decision Strategies Function of the Number of Mobile Sinks: In this series of experiments we studied the relative performance of various solutions to the event handling decision problem. From the two strategies we introduced, the FR (first responder) strategy requires ongoing information about the density and movement pattern of the visitors. In contrast, the CS (closest sink) strategy only requires a priori information about the layout of the attractions and paths of the park. As a baseline, we will use the RS (random sink) strategy, where the event is handled by a randomly assigned sink. All the strategies (including RS) require inter-sink coordination to avoid multiple assignments. We used CDPE for sink positioning with the number of mobile sinks varied between 1 and 20. The events are distributed according to the biased event distribution for all experiments. The results are the average of 20 experiments with different random seeds. We used the SLAW mobility model for this experiment.

Figure 15 shows the average event handling times and 


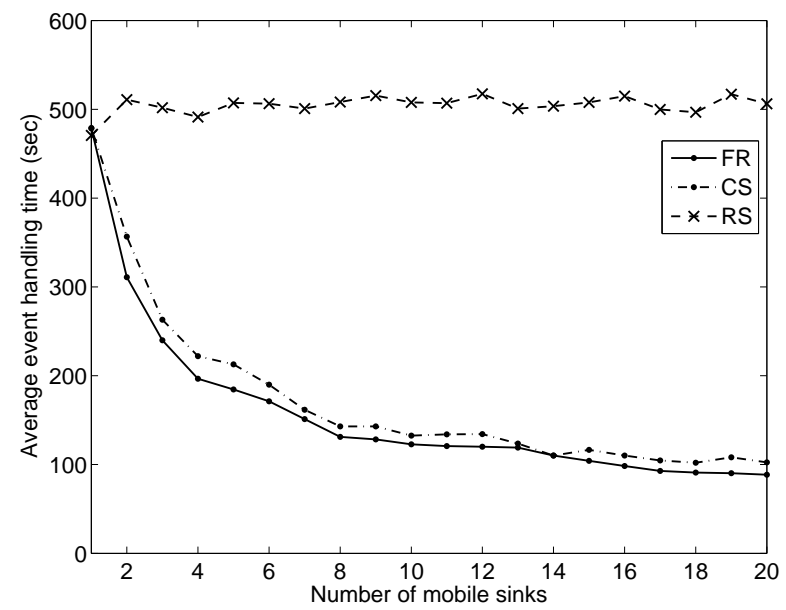

Fig. 15. Average event handling times for fastest responder (FR), closest sink (CS) and random sink (RS) event handling strategies function of the number of sinks

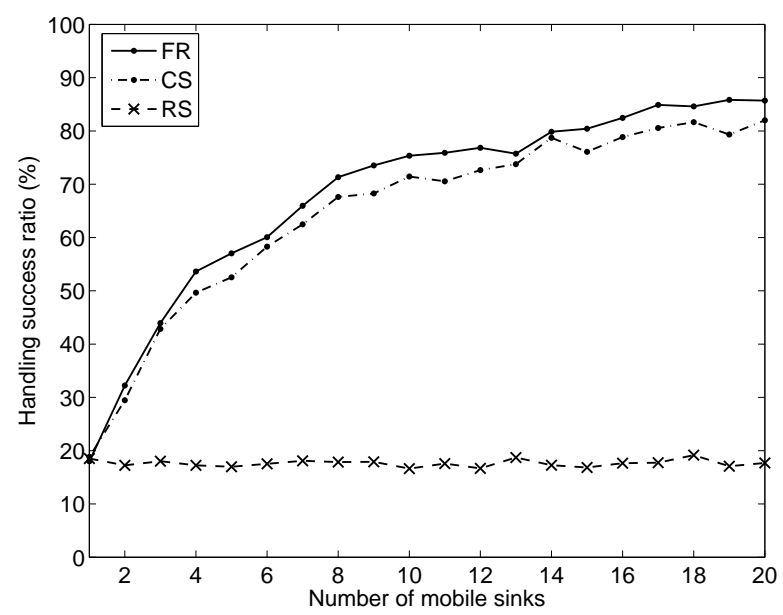

Fig. 16. Handling success ratios for fastest responder (FR), closest sink (CS) and random sink (RS) event handling strategies function of the number of sinks

Figure 16 the handling success ratios. The ranking is as expected, FR performing best, followed by CS and RS. While the performance of FR and CS increase with the number of sinks, the performance of RS does not. This is justified by the fact that with more sinks, it is more likely that FR and CS can find a sink which is close to the event. However, for RS which picks a sink randomly, the likelihood of being close or far is about the same independently of the number of sinks. While FR is consistently better than CS, their performance is relatively close: this additional benefit needs to be weighted against the expense of the crowd tracking sensor network.

5) The Impact of Event Distribution Types: In the last series of experiments, we study the effect of the different event distribution types outlined in Section III-D on the performance of the network. We used HSPE for 3 mobile sinks and the FR strategy for event handling and the TP visitor mobility model. Figure 17 shows the event handling times and Figure 18 the

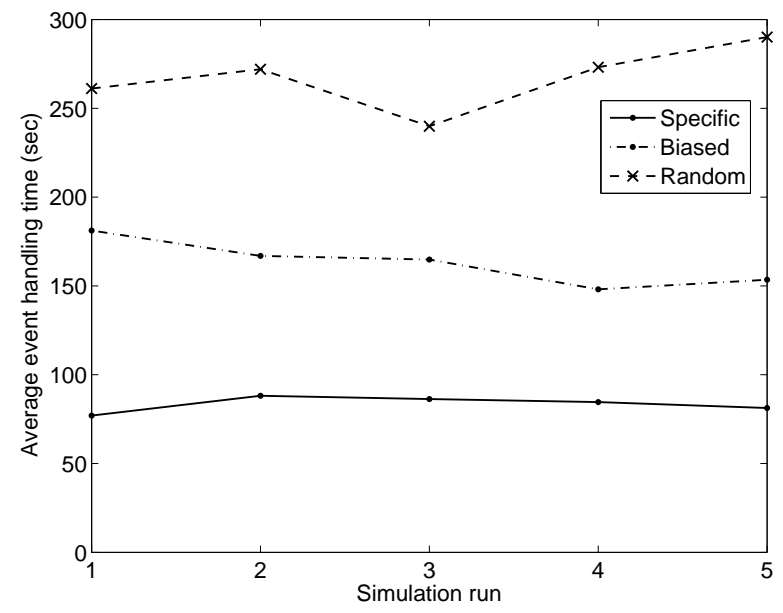

Fig. 17. Effect of event distribution types on event handling time.

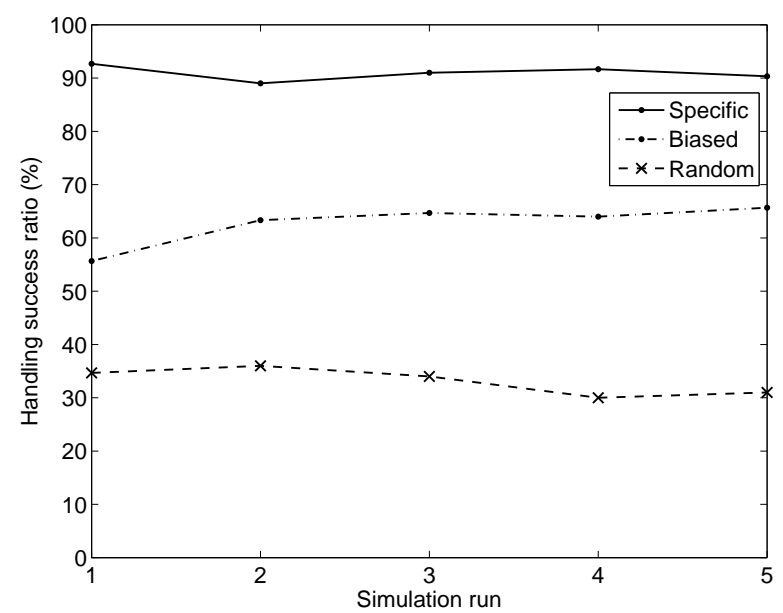

Fig. 18. Effect of event distribution types on handling success ratio.

handling success ratio for 5 different experiments. We find the event distribution has a very strong impact on the performance: the more random the distribution of the events, the lower the performance. The same setup which achieves $90 \%$ success rate on the scenario-specific distribution manages only $60 \%$ on the biased and about $35 \%$ at the random distribution. We conclude that stakeholders should perform careful initial studies on the distribution of events in the park, as the spatio-temporal distribution can have a critical impact on the event handling success, even if all the other parameters are the same.

\section{CONCLUSION}

In this paper, a wireless sensor network with mobile sinks model is proposed for event coverage in theme parks. A realistic human mobility model for theme parks (TP) is used to simulate the movement of theme park visitors. A dynamic directed graph model representing attractions, mobile sinks and dynamic events as the nodes and movement paths as the edges is proposed to model the environment. New strategies for sink positioning and event handling decision problems 
are introduced for the goal of event coverage. For mobile sink positioning, crowd density based probability estimation (CDPE) and hot-spot based probability estimation (HSPE) strategies are proposed. For event handling, we proposed fastest responder (FR) and closest sink (CS) strategies for both static and dynamic edge weights in the directed graph model.

The success of the model and strategies are evaluated through extensive simulations of different scenarios using TP mobility model and SLAW model. Furthermore, it is shown that using multiple mobile sinks has a significant advantage over using one mobile sink. We find that our model of wireless sensor network with multiple mobile sinks can be used for security and emergency applications in theme parks.

The techniques developed in this paper can be adapted to many applications which require the management of security in scenarios where a large number of people are visiting waypoints in a specific geographic area. Such applications include security in shopping malls, fairs and festivals. A related problem is the security in large transportation hubs such as airports and train stations.

\section{REFERENCES}

[1] G. Solmaz and D. Turgut, "Event coverage in theme parks using wireless sensor networks with mobile sinks," in Proc. of the IEEE ICC'13, June 2013, pp. 1522-1526.

[2] "Report on safety," Walt Disney Resorts and Parks, Tech. Rep., January 2008. [Online]. Available: http://thewaltdisneycompany.com/aboutdisney/security

[3] L. Guo, R. A. Beyah, and Y. Li, "SMITE: A stochastic compressive data collection protocol for mobile wireless sensor networks." in Proc. of the IEEE INFOCOM'11, April 2011, pp. 1611-1619.

[4] R. Pazzi, D. Zhang, A. Boukerche, and L. Mokdad, "E-TRAIL: energyefficient trail-based data dissemination protocol for wireless sensor networks with mobile sinks," in Proc. of the IEEE ICC'11, June 2011, pp. 1-5.

[5] X. Cheng, D.-Z. Du, L. Wang, and B. Xu, "Relay sensor placement in wireless sensor networks," Wireless Networks, vol. 14, no. 3, pp. 347355, June 2008.

[6] K. Xu, H. Hassanein, G. Takahara, and Q. Wang, "Relay node deployment strategies in heterogeneous wireless sensor networks," IEEE Transactions on Mobile Computing, vol. 9, no. 2, pp. 145 -159, February 2010.

[7] F. M. Al-Turjman, H. S. Hassanein, W. M. Alsalih, and M. Ibnkahla, "Optimized relay placement for wireless sensor networks federation in environmental applications," Wireless Communications and Mobile Computing, vol. 11, no. 12, pp. 1677-1688, December 2011.

[8] R. Sugihara and R. K. Gupta, "Path planning of data mules in sensor networks," ACM Transactions on Sensor Networks, vol. 8, no. 1, pp. 1-27, August 2011.

[9] N. Dimokas, D. Katsaros, L. Tassiulas, and Y. Manolopoulos, "High performance, low complexity cooperative caching for wireless sensor networks," Wireless Networks, vol. 17, no. 3, pp. 717-737, April 2011.

[10] G. Resta and P. Santi, "The fundamental limits of broadcasting in dense wireless mobile networks," Wireless Networks, vol. 18, no. 6, pp. 679695, August 2012.

[11] S. Basagni, A. Carosi, E. Melachrinoudis, C. Petrioli, and Z. M. Wang, "Controlled sink mobility for prolonging wireless sensor networks lifetime," Wireless Networks, vol. 14, no. 6, pp. 831-858, 2008.

[12] S. Basagni, A. Carosi, C. Petrioli, and C. A. Phillips, "Moving multiple sinks through wireless sensor networks for lifetime maximization," in MASS, Sep.-Oct. 2008, pp. 523-526.

[13] S. Basagni, A. Carosi, and C. Petrioli, "Heuristics for lifetime maximization in wireless sensor networks with multiple mobile sinks," in Proc. of the IEEE ICC'09, June 2009, pp. 1-6.

[14] S. Basagni, A. Carosi, C. Petrioli, and C. A. Phillips, "Coordinated and controlled mobility of multiple sinks for maximizing the lifetime of wireless sensor networks," Wireless Networks, vol. 17, no. 3, pp. 759778, April 2011.
[15] S. Basagni, M. Nati, C. Petrioli, and R. Petroccia, "ROME: Routing over mobile elements in WSNs," in Proc. of the IEEE GLOBECOM'2009, December 2009, pp. $1-7$.

[16] D. Vergados, A. Sgora, D. Vergados, and P. Chatzimisios, "Joint perflow scheduling and routing in wireless multihop networks," in Proc. of the ISCC'11, July 2011, pp. $213-217$.

[17] B. K. Haberman and J. W. Sheppard, "Overlapping particle swarms for energy-efficient routing in sensor networks," Wireless Networks, vol. 18, no. 4, pp. 351-363, May 2012.

[18] J. A. Sanchez, R. Marin-Perez, and P. M. Ruiz, "Beacon-less geographic multicast routing in a real-world wireless sensor network testbed," Wireless Networks, vol. 18, no. 5, pp. 565-578, July 2012.

[19] G. Kadas and P. Chatzimisios, "Collaborative efforts for safety and security in vehicular communication networks," in Proc. of the PCI'11, October 2011, pp. $117-121$.

[20] T. Camp, J. Boleng, and V. Davies, "A survey of mobility models for ad hoc network research," Wireless Communications and Mobile Computing, vol. 2, no. 5, pp. 483-502, September 2002.

[21] F. Bai and A. Helmy, "A survey of mobility models in wireless ad hoc networks," in Wireless Ad Hoc and Sensor Networks. Springer, October 2006, pp. 1-30.

[22] N. Aschenbruck, A. Munjal, and T. Camp, "Trace-based mobility modeling for multi-hop wireless networks," Computer Communications, vol. 34, no. 6, pp. 704-714, May 2011.

[23] I. Rhee, M. Shin, S. Hong, K. Lee, S. J. Kim, and S. Chong, "On the Lèvy-walk nature of human mobility," IEEE/ACM Transactions on Networking, vol. 19, no. 3, pp. 630-643, June 2011.

[24] K. Lee, S. Hong, S. J. Kim, I. Rhee, and S. Chong, "SLAW: self-similar least-action human walk," IEEE/ACM Transactions on Networking, vol. 20, no. 2, pp. 515-529, April 2012.

[25] V. Vukadinovic, F. Dreier, and S. Mangold, "Impact of human mobility on wireless ad hoc networking in entertainment parks," Ad Hoc Networks, June 2012

[26] B. Wang, H. B. Lim, and D. Ma, "A survey of movement strategies for improving network coverage in wireless sensor networks," Computer Communications, vol. 32, no. 13-14, pp. 1427-1436, August 2009.

[27] M. Di Francesco, S. K. Das, and G. Anastasi, "Data collection in wireless sensor networks with mobile elements: A survey," ACM Transactions on Sensor Networks, vol. 8, no. 1, pp. 1-31, August 2011.

[28] C. Zhu, L. Shu, T. Hara, L. Wang, S. Nishio, and L. T. Yang, "A survey on communication and data management issues in mobile sensor networks," Wireless Communications and Mobile Computing, pp. n/an/a, November 2011

[29] G. Anastasi, E. Borgia, M. Conti, and E. Gregori, "A hybrid adaptive protocol for reliable data delivery in WSNs with multiple mobile sinks," The Computer Journal, vol. 54, no. 2, pp. 213-229, February 2011.

[30] D. Turgut and L. Bölöni, "Three heuristics for transmission scheduling in sensor networks with multiple mobile sinks," in Proceedings of International Workshop on Agent Technology for Sensor Networks (ATSN-08), in conjunction with the Seventh Joint Conference on Autonomous and Multi-Agent Systems (AAMAS 2008), May 2008, pp. 1-8.

[31] D. Turgut and L. Bölöni, "Heuristic approaches for transmission scheduling in sensor networks with multiple mobile sinks," The Computer Journal, vol. 54, no. 3, pp. 332-344, March 2011.

[32] L. Bölöni and D. Turgut, "Should I send now or send later? A decisiontheoretic approach to transmission scheduling in sensor networks with mobile sinks," Special Issue of Wiley's Wireless Communications and Mobile Computing Journal (WCMC) on Mobility Management and Wireless Access, vol. 8, no. 3, pp. 385-403, March 2008.

[33] J. Luo, J. Panchard, M. Pirkowski, M. Grossglauser, and J. pierre Hubaux, "MobiRoute: routing towards a mobile sink for improving lifetime in sensor networks," in Proc. of the IEEE/ACM DCOSS'06, June 2006, pp. 480-497.

[34] S. A. Awwad, C. K. Ng, N. K. Noordin, and M. F. Rasid, "Cluster based routing protocol for mobile nodes in wireless sensor network," Wireless Personal Communications, vol. 61, no. 2, pp. 251-281, November 2011.

[35] H. Kanai, Y. Koizumi, H. Ohsaki, and M. Imase, "Gradient-based routing in delay tolerant mobile sensor networks incorporating node mobility," in Proc. of the CCNC'12, January 2012, pp. $235-239$.

[36] P. Zappi, E. Bales, J.-H. Park, W. Griswold, and T. Rosing, "The CitiSense air quality monitoring mobile sensor node," in Proc. of the IPSN'12 Workshop on Mobile Sensing, April 2012, pp. 1-5.

[37] M. Akbas, M. Brust, and D. Turgut, "SOFROP: self-organizing and fair routing protocol for wireless networks with mobile sensors and stationary actors," Special Issue of Elsevier's Computer Communications Journal on Specialized Routing and Node Placement Protocols for 
Emerging Wireless Ad Hoc Networks, vol. 34, no. 18, pp. 2135-2146, December 2011.

[38] R. Luo and O. Chen, "Mobile sensor node deployment and asynchronous power management for wireless sensor networks," IEEE Transactions on Industrial Electronics, vol. 59, no. 5, pp. 2377 -2385, May 2012.

[39] M. Akbas, G. Solmaz, and D. Turgut, "Actor positioning based on molecular geometry in aerial sensor networks," in Proc. of the IEEE ICC'12, June 2012, pp. 508-512.

[40] Z. Sun, I. Akyildiz, and G. Hancke, "Dynamic connectivity in wireless underground sensor networks," IEEE Transactions on Wireless Communications, vol. 10, no. 12, pp. 4334 -4344, December 2011.

[41] M. Erol-Kantarci, S. Oktug, L. Vieira, and M. Gerla, "Performance evaluation of distributed localization techniques for mobile underwater acoustic sensor networks," Ad Hoc Networks, vol. 9, no. 1, pp. 61-72, January 2011.

[42] M. Younis and K. Akkaya, "Strategies and techniques for node placement in wireless sensor networks: a survey," Ad Hoc Networks, vol. 6, no. 4, pp. 621-655, June 2008.

[43] Z. Vincze, K. Fodor, R. Vida, and A. Vidács, "Electrostatic modelling of multiple mobile sinks in wireless sensor networks," in Proc. of the IFIP Networking Workshop on Performance Control in Wireless Sensor Networks, May 2006, pp. 30-37.

[44] Y.-C. Wang, F.-J. Wu, and Y.-C. Tseng, "Mobility management algorithms and applications for mobile sensor networks," Wireless Communications and Mobile Computing, vol. 12, no. 1, pp. 7-21, January 2012.

[45] T. Melodia, D. Pompili, and I. F. Akyldiz, "Handling mobility in wireless sensor and actor networks," IEEE Transactions on Mobile Computing, vol. 9, no. 2, pp. 160-173, February 2010.

[46] Z. Vincze, D. Vass, R. Vida, A. Vidács, and A. Telcs, "Adaptive sink mobility in event-driven multi-hop wireless sensor networks," in Proc. of the InterSense'06, May 2006.

[47] C.-M. Angelopoulos, S. Nikoletseas, D. Patroumpa, and J. Rolim, "Coverage-adaptive random walks for fast sensory data collection," in Proc. of the ADHOC-NOW'10, August 2010, pp. 81-94.

[48] G. Solmaz, M. Akbas, and D. Turgut, "Modeling visitor movement in theme parks," in Proc. of the IEEE LCN'12, October 2012, pp. 36-43.

[49] M. Ester, H. P. Kriegel, J. Sander, and X. Xu, "A density-based algorithm for discovering clusters in large spatial databases with noise," in Proc. of the ICKDDM'96, August 1996, pp. 226-231.

[50] A. Boukerche, B. Turgut, N. Aydin, M. Ahmad, L. Bölöni, and D. Turgut, "Routing protocols in ad hoc networks: a survey," Computer Networks (Elsevier), vol. 55, no. 13, pp. 3032-3080, September 2011.

[51] "SEGWAY," http://www.segway.com.

[52] S. Wanhill, "Theme parks: Their development and operation," in Proc. of the CAUTHE'06, February 2006, pp. 1889-1921. 